Intern. J. Fuzzy Mathematical Archive

Vol. 11, No.1, 2016, 45-52

ISSN: 2320-3242 (P), 2320-3250 (online)

Published on 28 November 2016

www.researchmathsci.org

DOI: http://dx.doi.org/10.22457/ijfma.v11n1a7

\title{
Square Perfect Fuzzy Matching
}

\author{
P.Shakila Banu ${ }^{1}$ and R.Akilandeswari ${ }^{2}$
}

Department of Mathematics, Vellalar College for Women

Erode - 638 012, Tamilnadu, India

E-mail: ${ }^{1}$ shakimeeran10@ gmail.com;

Corresponding Author: ${ }^{2}$ akiladurai21@ gmail.com

Received 31 October 2016; accepted 16 November 2016

Abstract. Necessary and Sufficient conditions are given for a fuzzy graph on a cycle or a complete graph to be a square perfect fuzzy matching. As a consequence, it is shown that at a particular condition a square perfect fuzzy matching is not a $(2, k)$ regular fuzzy graph.

Keywords: Fuzzy graph, square fuzzy matching, square perfect fuzzy matching, regular fuzzy graph.

AMS Mathematics Subject Classification (2010): 03E72, 05C72, $05 \mathrm{C} 12$

\section{Introduction}

In 1965, Zadeh introduced the concept of a fuzzy set and fuzzy relation to representing the phenomena of uncertainly in real world problem. In 1975, Rosenfeld [4] introduced the concept of fuzzy graph Nagoor Gani and Radha [3] introduced on Regular fuzzy graph. Alison Northup [1] studied some properties on $(2, k)$ regular graph in her bachelor thesis. In [7], the authors introduced $d_{2}$ of a vertex in product graphs and also they discussed on $(2, k)$ regular and totally $(2, k)$ regular fuzzy graphs in [6]. Seethalakshmi and Gnanajothi studied about perfect fuzzy matching [8]. In this paper, we introduce square fuzzy matching and square perfect fuzzy matching. We derive the necessary and sufficient condition for the fuzzy graph on a cycle or a complete graph to be square perfect fuzzy matching. Also, we discuss some properties of square perfect fuzzy matching with examples.

\section{Preliminaries}

In this section, some basic definitions are given.

Definition 2.1. [4] A fuzzy graph $G$ is a pair of function $G:(\sigma, \mu)$ where $\sigma$ is a fuzzy subset of a non empty set $V$ and $\mu$ is a symmetric fuzzy relation on $\sigma$. The underlying crisp graph of $G:(\sigma, \mu)$ denoted by $G^{*}:(V, E)$ where $E \subseteq V \times V$. A fuzzy graph $G$ is complete if $\mu(u, v)=\mu(u v)=\sigma(u) \wedge \sigma(v)$ for all $u, v \in E$ where $u v$ denotes the edge between $u$ and $v$.

Definition 2.2. [3] Let $G$ : $(\sigma, \mu)$ be a fuzzy graph. The degree of a vertex $u$ is $d(u)=$ $\sum_{u \neq v} \mu(u, v)$. Since $\mu(u v)>0$ for $u v \in E$ and $\mu(u v)=0$ for uv $\notin E$, this is equivalent 
P.Shakila Banu and R.Akilandeswari

to $d(u)=\sum_{u v \in E} \mu(u, v)$. The minimum degree of $G$ is $\delta(G)=\wedge\{d(u) / u \in V\}$. The maximum degree of $G$ is $\Delta(G)=\vee\{d(u) / u \in V\}$.

Definition 2.3. [7] For a given graph $G$, the $d_{2}$ degree of a vertex $u$ in $G$, denoted by $d_{2}(u)$ means number of vertices at a distance two away from $u$.

Definition 2.4. [6] Let $G$ : $(\sigma, \mu)$ be a fuzzy graph. The $d_{2}$ degree of a vertex $u$ is $d_{2}(u)=$ $\sum_{u \neq v} \mu^{2}(u, v)$, where $\mu^{2}(u, v)=\left\{\mu\left(u, u_{1}\right) \wedge \mu\left(u_{1}, v\right)\right.$. Also $\mu(u v)=0$, for uv $\notin E$. The minimum $d_{2}$ degree of $G$ is $\delta(G)=\wedge\left\{d_{2}(u) / u \in V\right\}$. The maximum $d_{2}$ degree of $G$ is $\Delta(G)=\vee\left\{d_{2}(u) / u \in V\right\}$.

Definition 2.5. [7] A graph $G$ is said to be $(2, k)$ regular, $\left(d_{2}\right.$ - regular) if $d_{2}(u)=k$, for all $u$ in $G$.

Definition 2.6. [3] Let $G:(\sigma, \mu)$ be a fuzzy graph on $G^{*}:(V, E)$. If $d_{G}(u)=k$ for all $u \in V$, (i.e.,) if each vertex has same degree $k$, then $G$ is said to be a regular fuzzy graph of degree $k$ or a $k$ regular fuzzy graph.

Definition 2.7. [6] Let $G:(\sigma, \mu)$ be a fuzzy graph on $G^{*}:(V, E)$. If $d_{2}(u)=k$ for all $u \in V$, then $G$ is said to be $(2, k)$ regular fuzzy graph.

\section{Square perfect fuzzy matching}

Definition 3.1. Let $G:(\sigma, \mu)$ be a fuzzy graph on $G:(V, E)$. A subset $\mathrm{M}$ of $\mathrm{E}$ is called a square fuzzy matching if for each vertex $u$, we have $\sum_{v \in V} \mu^{2}(u, v) \leq \sigma(u)$.

Example 3.2. Let $G:(\sigma, \mu)$ be a fuzzy graph on the cycle $G^{*}:(V, E)$ where $V=$ $\left\{v_{1}, v_{2}, v_{3}, v_{4}\right\}$ and $E=\left\{e_{1}, e_{2}, e_{3}, e_{4}\right\}$ with $e_{1}=v_{1} v_{2}, e_{2}=v_{2} v_{3}, e_{3}=v_{3} v_{4}, e_{4}=$ $v_{4} v_{1} . \sigma\left(v_{1}\right)=0.7, \sigma\left(v_{2}\right)=0.5, \sigma\left(v_{3}\right)=0.4, \sigma\left(v_{4}\right)=0.7$. $\mu\left(e_{1}\right)=0.3, \mu\left(e_{2}\right)=0.4, \mu\left(e_{3}\right)=0.2, \mu\left(e_{4}\right)=0.5$

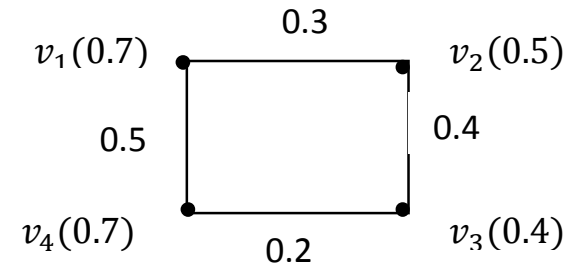

Figure 1:

$$
\begin{aligned}
& \sum_{\substack{v_{2} \in V \\
\left(v_{1}, v_{2}\right) \in M}} \mu^{2}\left(v_{1}, v_{2}\right)=\sum_{v_{2} \in V}\left(\mu\left(v_{1}, u\right) \wedge \mu\left(u, v_{2}\right)\right)=0.3 \wedge 0.4+0.2 \wedge 0.5 \\
& \sum_{\substack{v_{3} \in V \\
\left(v_{2}, v_{3}\right) \in M}} \mu^{2}\left(v_{2}, v_{3}\right)=0.3+0.2=0.5 \leq \sigma\left(v_{1}\right)
\end{aligned}
$$


Square Perfect Fuzzy Matching

$$
\begin{gathered}
\sum_{\substack{v_{4} \in V \\
\left(v_{3}, v_{4}\right) \in M}} \mu^{2}\left(v_{3}, v_{4}\right)=0.2 \wedge 0.5+0.3 \wedge 0.4=0.2+0.3=0.5 \$ \sigma\left(v_{3}\right) \\
\sum_{\substack{v_{1} \in V \\
\left(v_{4}, v_{1}\right) \in M}} \mu^{2}\left(v_{4}, v_{1}\right)=0.5 \wedge 0.3+0.2 \wedge 0.4=0.3+0.2=0.5 \leq \sigma\left(v_{4}\right)
\end{gathered}
$$

Thus $M=\left\{e_{1}, e_{2}, e_{4}\right\}$ is a square fuzzy matching in $G$.

Definition 3.3. A square fuzzy matching $M$ is called a square perfect fuzzy matching if $\sum_{v \in V} \mu^{2}(u, v)=\sigma(u)$.

Definition 3.4. Let $G:(\sigma, \mu)$ be a fuzzy graph and $\mathrm{M}$ be a square fuzzy matching. Then square fuzzy matching number $\Gamma(G)$ is defined to be $\Gamma(G)=\sum_{\substack{v \in M) \in M \\(u, v) \in M}} \mu^{2}(u, v)$.

Example 3.5. In example 3.2, $\Gamma(G)=1.5$.

Theorem 3.6. Let $G:(\sigma, \mu)$ be a fuzzy graph on the cycle $G^{*}:(V, E)$. Then $\sigma(u)=k$ is a constant function for all $u \in V$ and $\mu(u, v)=\frac{k}{2}$ for all $(u, v) \in E$ if and only if the following statement are equivalent

(i) $\mathrm{E}$ is a square perfect fuzzy matching

(ii) $(2, k)$ regular fuzzy graph.

Proof: Suppose that $\sigma$ is a constant function. Let $\sigma(u)=k$ is a constant, for all $u \in V$ and $\mu(u, v)=\frac{k}{2}$ for all $(u, v) \in E$. Assume that $\mathrm{G}$ is a $(2, k)$ regular fuzzy graph on the cycle $G^{*}:(V, E)$. Then $d_{2}(u)=k$. By definition of $d_{2}$ - degree of a vertex in fuzzy graph ie., $\sum \mu^{2}(u, v)=d_{2}(u)$

$\Rightarrow \sum \mu^{2}(u, v)=k$ for all $u \in V$. Since $G$ is a $(2, k)$ regular fuzzy graph.

$\Rightarrow \sum \mu^{2}(u, v)=\sigma(u)$ for all $u \in V$.

Each vertex of $u$ is satisfies the square perfect fuzzy matching in $G$.

Thus (ii) $\Rightarrow$ (i)

Now, suppose that $\mathrm{E}$ is a square perfect fuzzy matching on $G$. Since $G$ is a fuzzy graph on the cycle and only two edges are incident with each vertex for cycles, for any vertex $u \in V$.

$\Rightarrow \sum_{\substack{v \in V \\(u, v) \in M}} \mu^{2}(u, v)$ where $v, w \in V=\sigma(u)$.

$\Rightarrow \sum_{\substack{v \in V \\(u, v) \in M}} \mu^{2}(u, v)=k$ for all $u \in V$.

$\Rightarrow d_{2}(u)=\sum_{(u, v) \in M}^{v \in V} \mu^{2}(u, v)=k$ for all $u \in V$.

Hence $G$ is a $(2, k)$ regular fuzzy graph on cycle. Thus (i) $\Rightarrow$ (ii). Therefore, (i) $\Leftrightarrow$ (ii)

The converse parts holds trivially.

Example 3.7. Let $G:(\sigma, \mu)$ be a fuzzy graph on the cycle $G^{*}:(V, E)$ where $\mathrm{V}=$ $\left\{v_{1}, v_{2}, v_{3}, v_{4}\right\}$ and $\mathrm{E}=\left\{e_{1}, e_{2}, e_{3}, e_{4}\right\}$ with $e_{1}=v_{1} v_{2}, e_{2}=v_{2} v_{3}, e_{3}=v_{3} v_{4}, e_{4}=$ $v_{4} v_{1}$.

$\sigma\left(v_{1}\right)=0.8, \sigma\left(v_{2}\right)=0.8, \sigma\left(v_{3}\right)=0.8, \sigma\left(v_{4}\right)=0.8$

$\mu\left(e_{1}\right)=0.4, \mu\left(e_{2}\right)=0.4, \mu\left(e_{3}\right)=0.4, \mu\left(e_{4}\right)=0.4$. 
P.Shakila Banu and R.Akilandeswari

$$
\begin{aligned}
& 0.4 \\
& v_{1}(0.8) \int_{0.4}^{v_{2}(0.8)} \\
& v_{4}(0.8) \quad 0.4 \quad v_{3}(0.8) \\
& \sum_{\substack{v_{2} \in V \\
\left(v_{1}, v_{2}\right) \in M}} \mu^{2}\left(v_{1}, v_{2}\right)=\sum_{v_{2} \in V}\left(\mu\left(v_{1}, u\right) \wedge \mu\left(u, v_{2}\right)\right) \\
& =0.4 \wedge 0.4+0.4 \wedge 0.4=0.4+0.4=0.8=\sigma\left(v_{1}\right) \\
& \sum_{v_{3} \in V} \mu^{2}\left(v_{2}, v_{3}\right)=0.4 \wedge 0.4+0.4 \wedge 0.4=0.4+0.4=0.8=\sigma\left(v_{2}\right) \\
& \sum_{v_{4} \in V}^{\left(v_{2}, v_{3} \in M\right.} \mu^{2}\left(v_{3}, v_{4}\right)=0.4 \wedge 0.4+0.4 \wedge 0.4=0.4+0.4=0.8=\sigma\left(v_{3}\right) \\
& \sum_{\substack{v_{1} \in V \\
\left(v_{4}, v_{1}\right) \in M}}^{\left(v_{3}, v_{4}\right) \in M} \mu^{2}\left(v_{4}, v_{1}\right)=0.4 \wedge 0.4+0.4 \wedge 0.4=0.4+0.4=0.8=\sigma\left(v_{4}\right)
\end{aligned}
$$

Hence $G$ is a square perfect fuzzy matching and also $(2, k)$ regular fuzzy graph.

Example 3.8. In example 3.7, $\Gamma(G)=3.2$.

Theorem 3.9. Let $\mathrm{G}:(\sigma, \mu)$ be a $(2, k)$ regular fuzzy graph on the cycle $G^{*}:(V, E)$. If $\sigma(u)=k$ which is a constant function for all $u \in V$ and $\mu(u, v)=c$ where $c \leq k$ and $c \neq \frac{k}{2}$ for all $(u, v) \in E$. Then $\mathrm{E}$ is not a square perfect fuzzy matching on $G$.

Proof: Suppose that $\mathrm{G}$ is a fuzzy graph on the cycle and only two edges are incident with each vertex for cycles, for any vertex $u \in V$.

$$
\begin{aligned}
\Rightarrow \quad \sum_{(u, v) \in M} \mu^{2}(u, v) \text { where } v, w \in V=\mu^{2}(u, v)+\mu^{2}(v, w) \\
=\mu\left(u, u_{1}\right) \wedge \mu\left(u_{1}, v\right)+\mu\left(v, u_{n}\right) \wedge \mu\left(u_{n}, w\right) \\
=c \wedge c+c \wedge c=c+c=2 c \leq k \\
=c \leq \frac{k}{2}, \text { but } c \neq \frac{k}{2} \\
=c<k / 2 .
\end{aligned}
$$

Similarly $c>k / 2$

$\Rightarrow \sum_{\substack{v \in V \\(u, v) \in M}} \mu^{2}(u, v) \neq k$ for all $u \in V$

$\Rightarrow \sum_{\substack{v \in V \\(u, v) \in M}} \mu^{2}(u, v) \neq \sigma(u)$ for all $u \in V$.

Hence, E is not a square perfect fuzzy matching on $G$.

Remark 3.10. The condition $\mu(u, v)=k / 2$ is essential in theorem 3.6.This is illustrate with the following example. 
Square Perfect Fuzzy Matching

Example 3.11. Let $G:(\sigma, \mu)$ be a fuzzy graph on the cycle $G^{*}:(\mathrm{V}, \mathrm{E})$ where $\mathrm{V}=$ $\left\{v_{1}, v_{2}, v_{3}, v_{4}\right\}$ and $\mathrm{E}=\left\{e_{1}, e_{2}, e_{3}, e_{4}\right\}$ with $e_{1}=v_{1} v_{2}, e_{2}=v_{2} v_{3}, e_{3}=v_{3} v_{4}, e_{4}=$ $v_{4} v_{1}$.

$\sigma\left(v_{1}\right)=0.8, \sigma\left(v_{2}\right)=0.8, \sigma\left(v_{3}\right)=0.8, \sigma\left(v_{4}\right)=0.8 ; \mu\left(e_{1}\right)=0.5, \mu\left(e_{2}\right)=0.5$ $, \mu\left(e_{3}\right)=0.5, \mu\left(e_{4}\right)=0.5$

$$
\begin{aligned}
& \sum_{v_{2} \in V} \mu^{2}\left(v_{1}, v_{2}\right)=\sum_{v_{2} \in V}\left(\mu\left(v_{1}, u\right) \wedge \mu\left(u, v_{2}\right)\right)=0.5 \wedge 0.5+0.5 \wedge 0.5 \\
& \sum_{\substack{v_{3} \in V \\
\left(v_{2}, v_{3}\right) \in M}} \mu^{2}\left(v_{2}, v_{3}\right)=0.5+0.5=1 \neq \sigma\left(v_{1}\right) \\
& \sum_{\substack{v_{4} \in V \\
\left(v_{3}, v_{4}\right) \in M}} \mu^{2}\left(v_{3}, v_{4}\right)=0.5 \wedge 0.5+0.5 \wedge 0.5=0.5+0.5=1 \neq \sigma\left(v_{2}\right) \\
& \sum_{\substack{v_{1} \in V \\
\left(v_{4}, v_{1}\right) \in M}} \mu^{2}\left(v_{4}, v_{1}\right)=0.5 \wedge 0.5+0.5 \wedge 0.5=0.5+0.5=1 \neq \sigma\left(v_{3}\right) \\
& v_{2}
\end{aligned}
$$
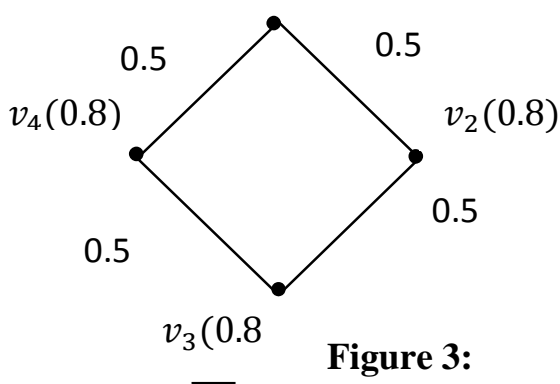

Hence $G$ is not a square perfect fuzzy matching.

Theorem 3.12. Let $G:(\sigma, \mu)$ be a square perfect fuzzy matching on the cycle $G^{*}:(V, E)$ of length $\geq 5$. If $\sigma\left(u_{i}\right)=\left\{\begin{array}{cl}\frac{3 k}{4} & , i=1,2, n-1, n \\ k & , i=3,4, \ldots, n-2\end{array}\right.$ for all $u \in V$ and $\mu\left(e_{i}\right)=\left\{\begin{array}{l}\frac{k}{2}, i=1,2, \ldots, n-1 \\ \frac{k}{4}, \quad i=n\end{array}\right.$

for all $(u, v) \in E$. Then $G$ is not a $(2, k)$ regular fuzzy graph.

Proof: Let $G:(\sigma, \mu)$ be a square perfect fuzzy matching on the cycle on $G^{*}:(V, E)$ is any cycle of length $\geq 5$. Let $e_{1}, e_{2}, \ldots, e_{n}$ be edges of a cycle of $G^{*}$ in that order.

$$
\begin{gathered}
d_{2}(u)=\sum_{\substack{v \in V \\
(u, v) \in M}} \mu^{2}(u, v) \\
d_{2}\left(u_{1}\right)=\mu\left(e_{1}\right) \wedge \mu\left(e_{2}\right)+\mu\left(e_{n}\right) \wedge \mu\left(e_{n-1}\right)=\frac{k}{2} \wedge \frac{k}{2}+\frac{k}{4} \wedge \frac{k}{2}=\frac{3 k}{4}=\sigma\left(u_{1}\right) \\
d_{2}\left(u_{2}\right)=\mu\left(e_{1}\right) \wedge \mu\left(e_{n}\right)+\mu\left(e_{2}\right) \wedge \mu\left(e_{3}\right)=\frac{k}{2} \wedge \frac{k}{4}+\frac{k}{2} \wedge \frac{k}{2}=\frac{k}{4}+\frac{k}{2}=\frac{3 k}{4}=\sigma\left(u_{2}\right) .
\end{gathered}
$$


P.Shakila Banu and R.Akilandeswari

For $i=3,4, \ldots, n-2$

$$
\begin{aligned}
& d_{2}\left(u_{i}\right)=\mu\left(e_{i-1}\right) \wedge \mu\left(e_{i-2}\right)+\mu\left(e_{i}\right) \wedge \mu\left(e_{i+1}\right)=\frac{k}{2} \wedge \frac{k}{2}+\frac{k}{2} \wedge \frac{k}{2}=k=\sigma\left(u_{i}\right) \\
& d_{2}\left(u_{n-1}\right)=\mu\left(e_{n-2}\right) \wedge \mu\left(e_{n-3}\right)+\mu\left(e_{n-1}\right) \wedge \mu\left(e_{n}\right)=\frac{k}{2} \wedge \frac{k}{2}+\frac{k}{2} \wedge \frac{k}{4}=\frac{3 k}{4}= \\
& \sigma\left(u_{n-1}\right) \\
& d_{2}\left(u_{n}\right)=\mu\left(e_{1}\right) \wedge \mu\left(e_{n}\right)+\mu\left(e_{n-1}\right) \wedge \mu\left(e_{n-2}\right)=\frac{k}{2} \wedge \frac{k}{4}+\frac{k}{2} \wedge \frac{k}{2}=\frac{3 k}{4}=\sigma\left(u_{n}\right) .
\end{aligned}
$$

Hence $G$ is not a $(2, k)$ regular fuzzy graph.

Example 3.13. Let $G:(\sigma, \mu)$ be a fuzzy graph on the cycle $G^{*}:(\mathrm{V}, \mathrm{E})$ where $\mathrm{V}=$ $\left\{v_{1}, v_{2}, v_{3}, v_{4}, v_{5}\right\}$ and $\mathrm{E}=\left\{e_{1}, e_{2}, e_{3}, e_{4}, e_{5}\right\}$ with $e_{1}=v_{1} v_{2}, e_{2}=v_{2} v_{3}, e_{3}=$ $v_{3} v_{4}, e_{4}=v_{4} v_{5}, e_{5}=v_{5} v_{1}$.$$
\sigma\left(v_{1}\right)=0.6, \sigma\left(v_{2}\right)=0.6, \sigma\left(v_{3}\right)=0.8, \sigma\left(v_{4}\right)=0.6, \sigma\left(v_{5}\right)=0.6
$$$$
\mu\left(e_{1}\right)=0.4, \mu\left(e_{2}\right)=0.4, \mu\left(e_{3}\right)=0.4, \mu\left(e_{4}\right)=0.4, \mu\left(e_{5}\right)=0.2 \text {. }
$$$$
\sum_{\substack{v_{2} \in V \\\left(v_{1}, v_{2}\right) \in M}} \mu^{2}\left(v_{1}, v_{2}\right)=\sum_{v_{2} \in V}\left(\mu\left(v_{1}, u\right) \wedge \mu\left(u, v_{2}\right)\right)=0.4 \wedge 0.4+0.2 \wedge 0.40 .6=\sigma\left(v_{1}\right)
$$

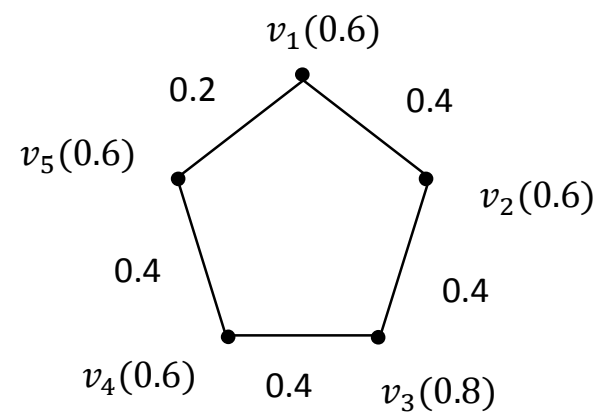

Figure 4:

$$
\begin{aligned}
& \sum_{\substack{v_{3} \in V \\
\left(v_{2}, v_{3}\right) \in M}} \mu^{2}\left(v_{2}, v_{3}\right)=0.4 \wedge 0.4+0.4 \wedge 0.2=0.4+0.2=0.6=\sigma\left(v_{2}\right) \\
& \sum_{\substack{v_{4} \in V \\
\left(v_{3}, v_{4}\right) \in M}} \mu^{2}\left(v_{3}, v_{4}\right)=0.4 \wedge 0.4+0.4 \wedge 0.4=0.4+0.4=0.8=\sigma\left(v_{3}\right) \\
& \sum_{\substack{v_{5} \in V \\
\left(v_{4}, v_{5}\right) \in M}}^{2} \mu^{2}\left(v_{4}, v_{5}\right)=0.4 \wedge 0.2+0.4 \wedge 0.4=0.2+0.4=0.6=\sigma\left(v_{4}\right) \\
& \sum_{\substack{v_{1} \in V \\
\left(v_{5}, v_{1}\right) \in M}} \mu^{2}\left(v_{5}, v_{1}\right)=0.2 \wedge 0.4+0.4 \wedge 0.4=0.2+0.4=0.6=\sigma\left(v_{5}\right)
\end{aligned}
$$

Hence $G$ is not a $(2, k)$ regular fuzzy graph.

Theorem 3.14. Let $\mathrm{G}:(\sigma, \mu)$ be a fuzzy graph on complete graph $K_{n}$ on $G:(V, E)$.If $\sigma(u)=k$ which is a constant function for all $u \in V$ and $\mu(u, v)=\left[\frac{k}{n}\right]=k_{1}$ for all $(u, v) \in E$ on the cycle $C_{n}$ and $\mu(u, v)=\frac{k-2 k_{1}}{n(n-3)}$ for all interior edges $(u, v) \in E$. Then E is a square perfect fuzzy matching on $G$. 


\section{Square Perfect Fuzzy Matching}

Proof: Let $\mathrm{G}:(\sigma, \mu)$ be any fuzzy graph on complete graph $K_{n}$, two edges are incident with each vertex of the cycle and remaining (n-3) edges are incident with interior vertices. The two distance of $n(n-3)$ edges are some edge one is interior and another one is cycle (or) some two edges are interior.

$$
\text { Hence } \quad \begin{aligned}
\sum \underset{\substack{v \in V \\
(u, v) \in M}}{ } \mu^{2}(u, v)=\sum_{\substack{v \in V \\
(u, v) \in M}}(\mu(u, w) \wedge \mu(w, v)) \\
=2[\mu(u, w) \wedge \mu(w, v)]+(n-3) n[\mu(u, w) \wedge \mu(w, v)] \\
=2\left(k_{1} \wedge k_{1}\right)+n(n-3)\left(\frac{k-2 k_{1}}{n(n-3)} \wedge k_{1}\right) \\
=2 k_{1}+n(n-3)\left(\frac{k-2 k_{1}}{n(n-3)}\right)=2 k_{1}+k-2 k_{1}=k=\sigma(u) .
\end{aligned}
$$

Hence $\mathrm{E}$ is a square perfect fuzzy matching on $\mathrm{G}$.

Remark 3.15. The condition $\mu(u, v)=\frac{k-2 k_{1}}{n(n-3)}$ is essential in theorem 3.14. This is illustrate with the following example.

Example 3.16. Let $\mathrm{G}:(\sigma, \mu)$ be a fuzzy graph on complete graph $K_{n}$ where $\mathrm{V}=$ $\left\{v_{1}, v_{2}, v_{3}, v_{4}, v_{5}\right\}$ and $\mathrm{E}=\left\{e_{1}, e_{2}, e_{3}, e_{4}, e_{5}, e_{6}, e_{7}, e_{8}, e_{9}, e_{10}\right\}$ with $e_{1}=v_{1} v_{2}, e_{2}=$ $v_{2} v_{3}, e_{3}=v_{3} v_{4}, e_{4}=v_{4} v_{5}, e_{5}=v_{5} v_{1}, e_{6}=v_{1} v_{3}, e_{7}=v_{1} v_{4}, e_{8}=v_{2} v_{4}, e_{9}=$ $v_{2} v_{5}, e_{10}=v_{3} v_{5} . \sigma\left(v_{1}\right)=1, \sigma\left(v_{2}\right)=1, \sigma\left(v_{3}\right)=1, \sigma\left(v_{4}\right)=1, \sigma\left(v_{5}\right)=1$. $\mu\left(e_{1}\right)=0.2, \mu\left(e_{2}\right)=0.2, \mu\left(e_{3}\right)=0.2, \mu\left(e_{4}\right)=0.2, \mu\left(e_{5}\right)=0.2, \mu\left(e_{6}\right)=0.1$, $\mu\left(e_{7}\right)=0.1, \mu\left(e_{8}\right)=0.1, \mu\left(e_{9}\right)=0.1, \mu\left(e_{10}\right)=0.1$.

$0.2 \quad v_{2}(1)$

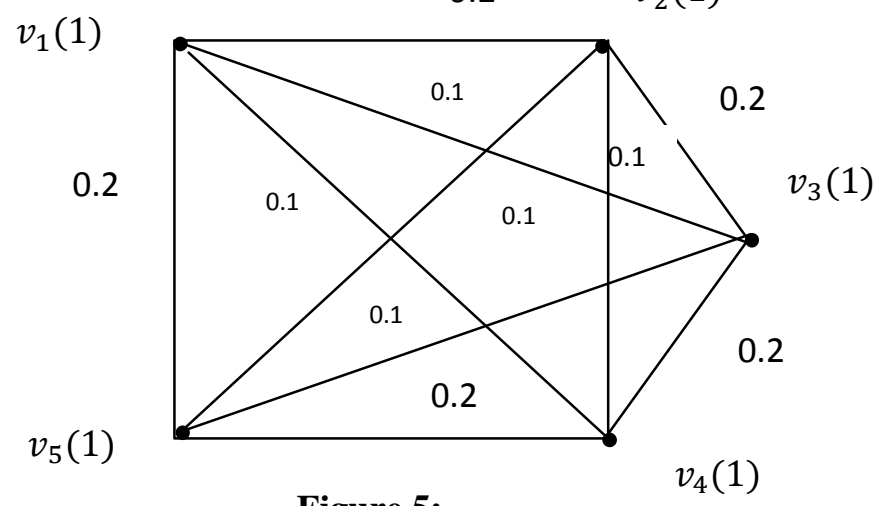

Figure 5:

$$
\begin{aligned}
& \sum_{\substack{v_{2} \in V \\
\left(v_{1}, v_{2}\right) \in M}} \mu^{2}\left(v_{1}, v_{2}\right)=\sum_{v_{2} \in V}\left(\mu\left(v_{1}, u\right) \wedge \mu\left(u, v_{2}\right)\right)=0.4+1=1.4 \neq \sigma\left(v_{1}\right) \\
& \sum_{\substack{v_{3} \in V \\
\left(v_{1}, v_{3}\right) \in M}} \mu^{2}\left(v_{1}, v_{3}\right)=2(0.2 \wedge 0.2)+5(5-3)[0.1]=0.4+1=1.4 \neq \sigma\left(v_{1}\right) \\
& \sum_{\substack{v_{4} \in V \\
\left(v_{1}, v_{4}\right) \in M}} \mu^{2}\left(v_{1}, v_{4}\right)=2(0.2 \wedge 0.2)+5(5-3)[0.1]=0.4+1=1.4 \neq \sigma\left(v_{1}\right) \\
& \sum_{\substack{v_{5} \in V \\
\left(v_{1}, v_{5}\right) \in M}} \mu^{2}\left(v_{1}, v_{5}\right)=2(0.2 \wedge 0.2)+5(5-3)[0.1]=0.4+1=1.4 \neq \sigma\left(v_{1}\right)
\end{aligned}
$$


P.Shakila Banu and R.Akilandeswari

$$
\begin{aligned}
& \sum_{\substack{v_{3} \in V \\
\left(v_{2}, v_{3}\right) \in M}} \mu^{2}\left(v_{2}, v_{3}\right)=2(0.2 \wedge 0.2)+5(5-3)[0.1]=0.4+1=1.4 \neq \sigma\left(v_{2}\right) \\
& \sum_{\substack{v_{4} \in V \\
\left(v_{2}, v_{4}\right) \in M}} \mu^{2}\left(v_{2}, v_{4}\right)=2(0.2 \wedge 0.2)+5(5-3)[0.1]=0.4+1=1.4 \neq \sigma\left(v_{2}\right) \\
& \sum_{\substack{v_{5} \in V \\
\left(v_{2}, v_{5}\right) \in M}} \mu^{2}\left(v_{2}, v_{5}\right)=2(0.2 \wedge 0.2)+5(5-3)[0.1]=0.4+1=1.4 \neq \sigma\left(v_{2}\right) \\
& \sum_{v_{4} \in V}^{\left(v_{3}, v_{4}\right) \in M} \mu^{2}\left(v_{3}, v_{4}\right)=2(0.2 \wedge 0.2)+5(5-3)[0.1]=0.4+1=1.4 \neq \sigma\left(v_{3}\right) \\
& \sum_{v_{5} \in V} \mu^{2}\left(v_{1}, v_{4}\right)=2(0.2 \wedge 0.2)+5(5-3)[0.1]=0.4+1=1.4 \neq \sigma\left(v_{3}\right) \\
& \sum_{\left.v_{3}, v_{5}\right) \in M} \mu^{2}\left(v_{4}, v_{5}\right)=2(0.2 \wedge 0.2)+5(5-3)[0.1]=0.4+1=1.4 \neq \sigma\left(v_{4}\right) \\
& \sum_{v_{5} \in V}^{\left(v_{4}, v_{5}\right) \in M} \\
& \sum_{v_{1} \in V} \mu^{2}\left(v_{5}, v_{1}\right)=2(0.2 \wedge 0.2)+5(5-3)[0.1]=0.4+1=1.4 \neq \sigma\left(v_{5}\right) \\
& \left(v_{5}, v_{1}\right) \in M
\end{aligned}
$$
matching in $G$.

Hence fuzzy graph on complete graph $K_{n}$ is not a square perfect fuzzy

\section{REFERENCES}

1. A.Northup, A Study of Semi-regular Graphs, Bachelors thesis, Stetson University (2002).

2. J.A.Bondy and U.S.R.Murty, Graph Theory with Applications, MacMillan, London (1979).

3. A.Nagoor Gani and K.Radha, On Regular Fuzzy Graphs, Journal of Physical Sciences, 12 (2008) 33-40.

4. A.Rosenfeld, Fuzzy Graphs, Fuzzy sets and their Applications, L.A.Zadeh, K.S. Fu, M.Shimura, Eds., Academic press (1975), 77-95.

5. N.R.Santhimaheswari and C.Sekar, (r, 2, (r-1) (r-1))-regular graphs, International Journal of Mathematics and Combinatorics, 4 (2012) 25-33.

6. N.R.Santhimaheswari and C.Sekar, On $(2, \mathrm{k})$-regular and totally $(2, \mathrm{k})$ - regular fuzzy graphs, International Journal of Mathematics and Soft Computing, 4(2) (2014)59-69.

7. N.R.Santhimaheswari and C.Sekar, On $\mathrm{d}_{2}$-of a vertex in product of graphs, ICODIMA (2013), Periyar Maniammai University, Thanjavur.

8. R.Seethalakshmi and R.B.Gnanajothi, A note on perfect fuzzy matching, International Journal of Pure and Applied Mathematics, 94(2) (2014) 155-161. 